Pacific Journal of Mathematic 


\title{
ON THE SEMIMETRIC ON A BOOLEAN ALGEBRA INDUCED BY A FINITELY ADDITIVE PROBABILITY MEASURE
}

\author{
Thomas E. Armstrong and Karel Prikry
}

\begin{abstract}
A finitely additive probability measure $\mu$ on a Boolean algebra $\mathscr{B}$ induces a semi-metric $d_{\mu}$ defined by $d_{\mu}(A, B)=$ $\mu(A \Delta B)$. When $\mathscr{B}$ is a $\sigma$-algebra and $\mu$ countably additive $\mathscr{B}$ is complete as is well known. The converse is shown to be true. More precisely, if $\mathscr{B}_{\mu}$ is the quotient of $\mathscr{B}$ via $\mu$-null sets then $\mathscr{B}_{\mu}$ is $d_{\mu}$-complete iff $\mu$ is countably additive on $\mathscr{B}_{\mu}$ and $\mathscr{B}_{\mu}$ is complete as a Boolean algebra. Furthermore $\mathscr{B}_{\mu}$ is $d_{\mu}$-complete iff every $\nu \ll \mu$ has a Hahn decomposition iff (when $\mathscr{B}$ is an algebra of sets) every $\nu \ll \mu$ has a $\mathscr{B}$-measurable Radon-Nikodym derivative. If $\mathscr{B}_{\mu}$ is not $d_{\mu}$-complete it is either meager in itself or fails to have the property of Baire in it's completion. Examples are given of both situations with the density character of $\mathscr{B}_{\mu}$ an arbitrary infinite cardinal number.
\end{abstract}

If $\mathscr{B}$ is a Boolean algebra with supremum $X$ and $\mu$ is a finitely additive probability measure on $\mathscr{B}$ (i.e., $\mu \in B A_{1}^{+}(\mathscr{B})$ ) there is a semi-metric $d_{\mu}$ on $\mathscr{B}$ given by $d_{\mu}(A, B)=\mu(A \Delta B)$ (where $\Delta$ denotes symmetric difference) for $\{A, B\} \subset \mathscr{B}$. Drewnowski [13] calls such semi-metrics Frechet-Nikodym semi-metrics. The metric space obtained by identifying $A$ and $B$ if $d_{\mu}(A, B)=0$ is the quotient Boolean algebra $\mathscr{B}_{\mu}=\mathscr{B} / \mathscr{N}_{\mu}$ where $\mathscr{N}_{\mu}$ is the ideal of $\mu$-negligible sets. We consider $\mu$ and $d_{\mu}$ to be defined on $\mathscr{B}_{\mu}$ in the usual manner so that $\mu(A \Delta B)=d_{\mu}(A, B)$ if $\{A, B\} \subset \mathscr{B}_{\mu}$. The operation of complementation is an isometry in $\mathscr{B}$ or $\mathscr{B}_{\mu}$ for $d_{\mu}$.

When $\mathscr{B}_{\mu}$ is $\sigma$-complete and $\mu$ is countably additive on $\mathscr{B}_{\mu}$ then $\mathscr{B}_{\mu}$ is complete both as a Boolean algebra and as a metric space. This fact has been very useful for analysts in the special case where $\mathscr{B}$ is a $\sigma$-algebra of subsets of $X$ and $\mu$ a countably additive measure on $\mathscr{B}$. In [12] it was asked to what extent this remains true if $\mu$ is only finitely additive. If $\mu$ is a $\{0,1\}$-valued measure on the Boolean algebra $\mathscr{B}$ then $\mathscr{B}_{\mu}$ is a two point space $\{\phi, X\}$ with $d_{\mu}(\phi, X)=1$. Thus, the theorem is true in this case. Of course, $\mu$ is then countably additive on $\mathscr{B}_{\mu}$. We may ask when $\mathscr{B}_{\mu}$ has an isolated point.

Proposition 1. $\mathscr{B}_{\mu}$ has an isolated point iff it is finite iff $\mu$ is a finite convex combination of $\{0,1\}$-valued measures. 
Proof. If $\mathscr{B}_{\mu}$ is finite it has a finite number of $\mu$-atoms and $\mu$ is a finite convex combination of $\{0,1\}$-valued measures. If $\mu$ isn't a finite convex combination of $\{0,1\}$-valued measures there is an infinite sequence $\left\{A_{n}\right\} \subset \mathscr{B}_{\mu} \backslash\{\phi\}$ with $\lim _{n \rightarrow \infty} \mu\left(A_{n}\right)=0$. If $A \in \mathscr{B}_{\mu}$ then $A \neq A \Delta A_{n}$ for large $n$ and $\lim _{n \rightarrow \infty} d_{\mu}\left(A, A \Delta A_{n}\right)=0$. Thus, $A$ isn't isolated. This suffices to establish the proposition.

Thus, except in trivial cases, $\mathscr{B}_{\mu}$ is an infinite perfect metric space. It turns out that the only time $\mathscr{B}_{\mu}$ is complete under $d_{\mu}$ is when $\mathscr{B}_{\mu}$ is complete as a Boolean algebra and $\mu$ is countably additive on $\mathscr{B}_{\mu}$.

Proposition 2. In order that $\mathscr{B}_{\mu}$ be a complete metric space under $d_{\mu}$ it is necessary and sufficient that $\mathscr{B}_{\mu}$ be a complete Boolean algebra and that $\mu$ be countably additive on $\mathscr{B}_{\mu}$.

Proof. First suppose that $\mathscr{B}_{\mu}$ isn't a complete Boolean algebra. Since $\mathscr{B}_{\mu}$ satisfies the countable chain condition it can't be a $\sigma$-complete Boolean algebra. Thus, there is an increasing sequence $\left\{A_{n}\right\} \subset$ $\mathscr{B}_{\mu}$ without a supremum in $\mathscr{B}_{\mu}$. Let $\lambda=\lim _{n \rightarrow \infty} \mu\left(A_{n}\right)$. We have $d_{\mu}\left(A_{n}, A_{n+k}\right)=\mu\left(A_{n+k} \backslash A_{n}\right) \leqq \lambda-\mu\left(A_{n}\right) \rightarrow 0$ as $n \rightarrow \infty$. Thus, $\left\{A_{n}\right\}$ is $d_{\mu}$-Cauchy. If $A \in \mathscr{B}_{\mu}$ were such that $\lim _{n \rightarrow \infty} d_{\mu}\left(A_{n}, A\right)=0$ then $\lim _{n \rightarrow \infty} \mu\left(A_{n} \backslash A\right)=0$ so $\mu\left(A_{n} \backslash A\right)=0$ for all $n$ hence $A_{n} \subset A$ for all $A$. From $\lim _{n \rightarrow \infty} \mu\left(A \backslash A_{n}\right)=0$ it would follow that $A=\sup _{n} A_{n}$ which is impossible. Thus, if $\mathscr{B}_{\mu}$ is $d_{\mu}$-incomplete it is incomplete as a Boolean algebra.

Now suppose that $\mathscr{B}_{\mu}$ is a complete Boolean algebra with $\mu$ not countably additive. There exists an increasing sequence $\left\{A_{n}\right\} \subset \mathscr{B}_{\mu}$ with union $A$ so that $\lim _{n \rightarrow \infty} \mu\left(A_{n}\right)=\lambda<\mu(A)$. Once again, $\left\{A_{n}\right\}$ must be $d_{\mu}$-Cauchy and if $C \in \mathscr{B}_{\mu}$ with $\lim _{n \rightarrow \infty} d_{\mu}\left(A_{n}, C\right)=0$ then $C=A$. Since $\lim _{n \rightarrow \infty} d_{\mu}\left(A_{n}, A\right)=\mu(A)-\lambda \neq 0, \mathscr{B}_{\mu}$ is $d_{\mu}$-incomplete. Thus, if $\mathscr{B}_{\mu}$ is $d_{\mu}$-complete then $\mu$ is countably additive on $\mathscr{B}_{\mu}$. This suffices to establish the proposition.

Plachky, [23] gives a characterization of extreme extensions $\nu$ of a finitely additive probability $\mu$ on $\mathscr{B}_{1}$ to $\mathscr{B}_{2}$. He denotes by $b a\left(\mathscr{B}_{1}, \mu, \mathscr{B}_{2}\right)$ all such extensions. We denote by $\xi b a\left(\mathscr{B}_{1}, \mu, \mathscr{B}_{2}\right)$ the extreme elements of the compact convex set $b a\left(\mathscr{B}_{1}, \mu, \mathscr{B}_{2}\right)$. In terms of the semi-metric $d_{\nu}$ elements $\nu$ of $\xi b a\left(\mathscr{B}_{1}, \mu, \mathscr{B}_{2}\right)$ are characterized by the condition that for all $A_{2} \in \mathscr{B}_{2}$ and $\varepsilon>0$ there is an $A_{1} \in \mathscr{B}_{1}$ with $d_{\nu}\left(A_{1}, A_{2}\right)<\varepsilon$. That is, $\nu \in \xi b a\left(\mathscr{B}_{1}, \mu, \mathscr{B}_{2}\right)$ iff $\mathscr{B}_{1}$ is $d_{\nu}$-dense in $\mathscr{B}{ }_{2}$.

CoRollary 2.1. Let $\mathscr{B}_{1} \subset \mathscr{B}_{2}$ be Boolean algebras and let $\mu$ be 
a probability measure with $\mathscr{B}_{1} d_{\mu}$-complete. For $\nu \in$ ba $\left(\mathscr{B}_{1}, \mu, \mathscr{B}_{2}\right)$ to be in $\xi b a\left(\mathscr{P}_{1}, \mu, \mathscr{B}_{2}\right)$ it is necessary and sufficient that for all $A_{2} \in \mathscr{B}_{2}$ there be an $A_{1} \in \mathscr{B}_{1}$ with $d_{\nu}\left(A_{1}, A_{2}\right)=0$. If $\nu \in b a\left(\mathscr{B}_{1}, \mu, \mathscr{B}_{2}\right)$ then $\mathscr{B}_{2}$ is $d_{\nu}$-complete.

Proof. It is only necessary to show that if $\nu \in \xi b a\left(\mathscr{B}_{1}, \mu, \mathscr{B}_{2}\right)$ and $A_{2} \in \mathscr{B}_{2}$ there is an $A_{1} \in \mathscr{B}_{1}$ with $d_{\nu}\left(A_{1}, A_{2}\right)=0$. By Plachky's condition we may construct a sequence $\left\{A^{n}\right\} \subset \mathscr{B}_{1}$ which $d_{\nu}$-converges to $A_{2}$. Any $d_{\mu}$-limit $A_{1}$ of this sequence will suffice.

Remarks. (1) Bogdan and Oberle in Proposition 1.1.1 of [9] obtain a result closely related to Proposition 2. M. Bhaskara Rao and K. P. S. Bhaskara Rao in [25] essentially obtain Propositions 1 and 2.

(2) Corollary 2.1 yields a method for obtaining noncountably additive $\mu$ with $\mathscr{B}_{\mu} d_{\mu}$-complete.

Recall that a finitely additive measure $\mu$ of bounded variation on a Boolean algebra $\mathscr{B}$ (i.e., $\mu \in B A(\mathscr{B})$ ) has a Hahn decomposition iff there is an $A \in \mathscr{B}$ so that $\mu(A)=\left\|\mu^{+}\right\|$and $\mu\left(A^{c}\right)=\left\|u^{-}\right\|$. Thus, $\mu^{+}(E)=\mu(A \cap E)$ and $\mu^{-}(E)=\mu\left(E \cap A^{c}\right)$ if $E \in \mathscr{B}$. Here, $\mu^{+}$and $\mu^{-}$are the positive and negative variations of $\mu$. $|\mu|=\mu^{+}+\mu^{-}$ is the total variation of $\mu$.

Proposition 3. Let $\mu$ be a probability measure on the algebra $\mathscr{B} . \mathscr{B}_{\mu}$ is $d_{\mu}$-complete iff every $\nu \in B A(\mathscr{B})$ with $|\nu|=\mu$ has a Hahn decomposition iff every $\nu \in B A(\mathscr{B})$ with $\nu \ll \mu$ has a Hahn decomposition.

Proof. If $\mu$ is countably additive on the complete algebra $\mathscr{B}_{\mu}$ then every $\nu \in B A(\mathscr{B})$ with $\nu \ll \mu$ is countably additive on $\mathscr{B}_{\mu}$ hence has a Hahn-decomposition in $\mathscr{B}_{\mu}$ and in $\mathscr{B}$ (we are using the $\varepsilon-\delta$ definition of absolute continuity $\ll$ as in [8]). Only the converse needs to be established.

We must show that if every $\nu \in B A(\mathscr{B})$ with $|\nu|=\mu$ has a Hahn-decomposition then $\mathscr{B}_{\mu}$ is $d_{\mu}$-complete. Suppose that $\mu$ isn't countably additive on $\mathscr{B}_{\mu}$. There exists $\left\{A_{n}\right\}$ an increasing sequence in $\mathscr{B}_{\mu}$ with supremum $X$ such that $0<\lim _{n \rightarrow \infty} \mu\left(A_{n}\right)=\lambda<1$. Let $\mu^{\prime}(A)=\lim _{n \rightarrow \infty} \mu\left(A \cap A_{n}\right)$ define $\mu^{\prime}(A)$ for $A \in \mathscr{B}_{\mu}$ so that $\mu^{\prime} \in B A^{+}\left(\mathscr{B}_{\mu}\right)$ hence $\mu^{\prime} \in B A^{+}(\mathscr{B})$. Let $\mu^{\prime \prime}=\mu-\mu^{\prime} \in B A^{+}(\mathscr{B})$. Let $\nu=\mu^{\prime}-\mu^{\prime \prime} \in$ $B A(\mathscr{B})$. Since $\mu^{\prime}$ and $\mu^{\prime \prime}$ may be verified to be singular, $\nu^{+}=\mu^{\prime}$, $\nu^{-}=\mu^{\prime \prime}$ and $|\nu|=\mu$. Let $A \in \mathscr{B} \mu$ be such that $\nu(A)=\nu^{+}(A)$ and $-\nu\left(A^{c}\right)=\nu^{-}(A)$. We have $\nu^{+}(A)=\mu^{\prime}(A)=\lim _{n \rightarrow \infty} \mu\left(A \cap A_{n}\right)=\left\|\mu^{\prime}\right\|=$ $\lambda$. Thus, $A_{n} \subset A$ for all $n$. Thus, $A=X$ and $\mu^{\prime \prime}=0$ which is impossible. Thus, $\mu$ must be countably additive on $\mathscr{B}_{\mu}$. 
If $\mathscr{B}_{\mu}$ isn't $\sigma$-complete there is an increasing sequence $\left\{A_{n}\right\}$ without a supremum. Define $\mu^{\prime}(A)=\lim _{n \rightarrow \infty} \mu^{\prime}\left(A_{n} \cap A\right)$ so that $\mu^{\prime} \epsilon$ $B A^{+}\left(\mathscr{B}_{\mu}\right)$ hence $\mu^{\prime} \in B A^{+}(\mathscr{B})$ let $\mu^{\prime \prime}=\mu-\mu^{\prime}$ and let $\nu=\mu^{\prime}-\mu^{\prime \prime}$. If $\mu^{\prime \prime}=0$ then $X=\sup _{n} A_{n}$ and if $\mu^{\prime}=0$ then $\phi=\sup _{n} A_{n}$ which are impossible. If $A \in \mathscr{B}_{\mu}$ is such that $\nu^{+}(E)=\nu(E \cap A)$ and $\nu^{-}(E)$ : $-\nu\left(E \cap A^{v}\right)$. Once again, $A$ would have to be $\sup _{n} A_{n}$ which is impossible. Since such an $A$ is guaranteed to exist $\mathscr{B}_{\mu}$ must be $\sigma$-complete hence complete.

$\mathscr{B}$ may be an algebra of subsets of $X$. This is the case if $X$ is the Stone space $X_{\mathscr{B}}$ of $\mathscr{B}$ and $\mathscr{B}$ is regarded as the clopen algebra of $X_{\mathscr{B}}$. If $\mu \in B A(\mathscr{B})$ one may integrate simple step functions $f=\sum_{i=1}^{n} \lambda_{i} \chi_{A_{i}}$ with $\left\{A_{1}, \cdots, A_{n}\right\}$ in the usual manner. One may integrate any $f$ which is the uniform limit of simple step functions as the limit of the integrals of the step functions. The totality of such $f$ will be called bounded $\mathscr{B}$-measurable functions. More generally $f: X \rightarrow[-\infty, \infty]$ is called $\mathscr{B}$-measurable iff $f \wedge n \vee(-m)$ is a bounded $\mathscr{B}$-measurable function for all integers $n, m \geqq 0$. One defines $\int f d \mu$, for any $\mathscr{B}$-measurable $f$, to be $\lim _{(m, n) \rightarrow(\infty, \infty)} \int f \wedge n \vee$ $(-m) d \mu$ provided this limit exists. For any $\mathscr{B}$-measurable $f$ on $X$ with $\int|f| d \mu<\infty$ one may define the measure $f \mu$ on $\mathscr{B}$ by the requirement that $(f \mu)(A)=\int f \chi_{A} d \mu$ for $A \in \mathscr{B}$. Then, $f \mu \in B A(\mathscr{B})$ and is absolutely continuous with respect to $\mu$. If $\mu \in B A^{+}(\mathscr{B})$ one has $(f \mu)^{+}=(f \vee 0) \mu,(f \mu)^{-}=-(f \wedge 0) \mu$ and $|f \mu|=|f| \mu$. If $g$ is $\mathscr{B}$-measurable and $\int g d(f \mu)$ exists it is $\int g f d \mu$. If $\nu \ll \mu \in B A^{+}(\mathscr{B})$ one says that $\nu$ has a Radon-Nikodym derivative, $f=d \nu / d \mu$, iff $f$ is $\mathscr{B}$-measurable with $\nu=f \mu$. When $\mu$ is a countably additive probability on the $\sigma$-complete $\mathscr{B}_{\mu}$ (i.e., when $\mathscr{B}_{\mu}$ is $d_{\mu}$-complete) every $\nu \ll \mu$ has a Radon-Nikodym derivative on $\mathscr{B}_{\mu}$ with respect to $\mu$ and on $\mathscr{B}$ if $\mu$ is countably additive on $\mathscr{B}$.

Proposition 4. Let $\mathscr{B}$ be a Boolean set algebra and let $\mu \epsilon$ $B A_{1}^{+}(\mathscr{B}) . \quad \mathscr{B}_{\mu}$ is $d_{\mu}$-complete iff every $\nu \ll \mu$ has a Radon-Nikodym derivative on $\mathscr{B}$ (hence on $\mathscr{B}_{\mu}$ ).

Proof. There is a Banach lattice isomorphism between the $M$ space of bounded $\mathscr{B}$-measurable functions on $X$ and the continuous functions on the Stone space $X_{\mathscr{B}}$. If the bounded $\mathscr{B}$-measurable $f$ on $X$ has corresponding to it $\tilde{f}$ and the finitely additive $p \in B A(\mathscr{B})$ has corresponding to it $\widetilde{p} \in \mathscr{L}\left(X_{\mathscr{G}}\right)$ under the Stone correspondence then $\int_{X} f d p=\int_{X \mathscr{f}} \tilde{f} d \tilde{p}$. For $\nu \in B A(\mathscr{B}), \nu=f \mu$ with $f$ bounded and $\mathscr{B}$-measurable iff $\tilde{\nu}=\tilde{f} \tilde{\mu}$ with $\tilde{f} \in C\left(X_{\mathscr{B}}\right)$. If $|\nu|=\mu$ then $|\tilde{f}| \tilde{\mu}=$ 
$|\tilde{\nu}|=\tilde{\mu}$ so $|\tilde{f}|=1$ on $\operatorname{supp}(\tilde{\mu})$. There is a clopen set $[A] \subset X_{\mathscr{\mathscr { C }}}$ corresponding to $A \in \mathscr{B}$ so that $\tilde{f}=\chi_{[A]}-\chi_{\left[A^{c}\right]}$ on $\operatorname{supp}(\tilde{\mu})$ consequently $\tilde{\nu}=\left(\chi_{[A]}-\chi_{\left[A^{c}\right]}\right) \tilde{\mu}$ and $\nu=\left(\chi_{A}-\chi_{A^{c}}\right) \mu$. Thus, $\nu$ has a Hahn-decomposition. Since $\nu$ was arbitrary with $|\nu|=\mu \mathscr{B}_{\mu}$ is $d_{\mu}$-complete by Proposition 3.

Now suppose that $\mathscr{B}_{\mu}$ is $d_{\mu}$-complete. If $\lambda^{-1} \mu \leqq \nu \leqq \lambda \mu$ for some $\lambda \in(0, \infty)$ then, on $X_{\mathscr{B} \mu}$, there is a Radon-Nikodym derivative $g$ for $\nu$ with respect to $\mu$ which is bounded and $\mathscr{B}_{\mu}$-measurable hence continuous $\left(\mathscr{B}_{\mu}\right.$ is considered to be the clopen algebra of $\left.X_{\mathscr{C}_{\mu}}\right)$. If $\tilde{\nu}$ and $\tilde{\mu}$ are the Radon measures on $X_{\mathscr{D}_{\mu}}$ corresponding to $\nu$ and $\mu$ we have $\tilde{\nu}=g \tilde{\mu}$. Extend $g$ continuously from $X_{\mathscr{A} \mu}$, considered as a closed subspace of $X_{\mathscr{G}}$, to a continuous function $f$ on $X_{\mathscr{B}}$. Then, $\tilde{\nu}=f \tilde{\mu}$ where $\{\tilde{\nu}, \tilde{\mu}\}$ are considered as Radon measures on $X_{\mathscr{B}} . f$ is $\mathscr{B}$-measurable on $X_{\mathscr{B}}$ hence is the uniform limit of simple step functions $\left\{f_{n}\right\}$. If $X=X_{\mathscr{A}}$ then $\nu=f \mu$. Otherwise $\left\{f_{n}\right\}$ corresponds to a uniformly convergent sequence $\left\{f_{n}^{\prime}\right\}$ of simple step functions on $X$ (where $f_{n}^{\prime}(x)=f_{n}(\hat{x})$ where $\hat{x} \in X_{\mathscr{B}}$ is the ultrafilter of supersets of $x$ in $\mathscr{B})$. Once again $\nu=f^{\prime} \mu$ where $f^{\prime}=\lim _{n \rightarrow \infty} f_{n}^{\prime}$.

If $\nu \ll \mu$ then $\nu$ is the limit in the variation norm of $\nu_{n}=\nu \wedge$ $(n \mu) \vee(-n \mu)$ as $n \rightarrow \infty$. We have $\nu_{n}=\nu_{n+k} \wedge(n \mu) \vee(-n \mu)$ for all $k>0$. Since $-n \mu \leqq \nu \leqq n \mu$ we have $\nu=f_{n} \mu$ and $f_{n}=f_{n+k} \wedge n \wedge-n$ for $k>0$ where $\left\{f_{n}\right\}$ are $\mathscr{B}$-measurable on $X$. Define $f(x)=f_{n}(x)$ if $f_{n+k}(x)=f_{n}(x)$ for all $k>0$. If $f(x)$ isn't defined either $f_{n}(x)=n$ for all $n$ or $f_{n}(x)=-n$. In the first case set $f(x)=\infty$ and in the second set $f(x)=-\infty$. Since $f \wedge n \vee-n=f_{n}$ is $\mathscr{B}$-measurable it follows that $f$ is $\mathscr{B}$-measurable. If $A \in \mathscr{B}$ then $\nu(A)=\lim _{n \rightarrow \infty} \nu_{n}(A)=$ $\lim _{n \rightarrow \infty} \int_{A} f_{n} d \mu=\int_{A} f d \mu$. Thus, $f=d \nu / d \mu$. This establishes the proposition.

REMARK. Since $\mathscr{B}_{\mu}$ remains unchanged if $\mathscr{B}$ is enlarged, and $\mu$ redefined, by only an enlargement of $\eta_{\mu}$ we may consider $\hat{\eta}_{\mu}$ the set of $A$ with $A \subset X$ such that for all $\varepsilon>0$ there is an $A^{\varepsilon} \in \mathscr{B}$ with $A \subset A^{\varepsilon}$ and $\mu\left(A^{\varepsilon}\right) \leqq \varepsilon$. Let $\mathscr{B} \Delta \hat{\eta}_{\mu}$ denote all sets $A^{\prime}$ in $X$ differing from an $A \in \mathscr{B}$ by an $N \in \hat{\eta}_{\mu}$. For such $A^{\prime}$ set $\mu\left(A^{\prime}\right)=\mu(A)$ so that $\hat{\eta}_{\mu}$ is the ideal of $\mu$-negligible sets in $\mathscr{B} \Delta \hat{\eta}_{\mu}$. Propositions 3 and 4 remain unchanged when $\mathscr{B}$ is replaced by $\mathscr{B} \Delta \hat{\eta}_{\mu}$.

In general $\mathscr{B}_{\mu}$ isn't complete under $d_{\mu}$ but its completion is easily identified.

Proposition 5. Let $\mu \in B A_{1}^{+}(\mathscr{B}), X_{\mathscr{B}}\left(X_{\mathscr{B} \mu}\right)$ be the Stone space

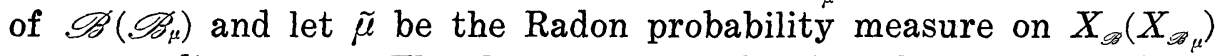
corresponding to $\mu$. The $d_{\mu}$-completion of $\mathscr{B}$ is the quotient of the Baire algebra, $\mathscr{B}^{0}$, of $X_{\mathscr{B}}\left(X_{\mathscr{B}}\right)$ modulo $\tilde{\mu}$-negligible sets (i.e., $\mathscr{B}_{\tilde{\mu}}^{0}$ ) 
under $d_{\mu}$.

Proof. It is easiest to work with $\mathscr{B}_{\mu}$ considered as the clopen algebra of $X_{\mathscr{B}_{\mu}}$. Then $\mathscr{B}_{\mu} \subset \mathscr{B}^{0}$ and the metric $d_{\mu}$ on $\mathscr{B}_{\mu}$ is the same as is induced by the semi-metric $d_{\tilde{\mu}}$. As a result $\mathscr{B}_{\mu}$ is isometric to a subset of the $d_{\tilde{\mu}}$-complete $\mathscr{B}_{\mu}{ }^{0}$. Since $\mathscr{B}^{0}$ is the monotone sequential closure of $\mathscr{B}_{\mu}$ it follows that $\mathscr{B}_{\mu}$ is $d_{\mu}$-dense in $\mathscr{B}^{0}$ hence in $\mathscr{B}_{\tilde{\mu}}^{0}$. Thus, $\mathscr{B}_{\tilde{\mu}}^{0}$ must be the completion of $\mathscr{B}_{\mu}$.

Remark. Proposition 2 is an immediate corollary of Proposition 5.

One may extend $\mu$ defined on the algebra, $\mathscr{B}$ of subsets of $X$, not just to $\mathscr{B} \Delta \hat{\eta}_{\mu}$ but to an even larger algebra $\hat{\mathscr{B}}_{\mu}$ of subsets of $X$ in a unique manner. $\hat{\mathscr{B}}_{\mu}$ is the $\mu$-completion of $\mathscr{B}$ and consists of those sets $E \subset X$ so that $\mu^{*}(E)=\inf \{\mu(A): E \subset A \in \mathscr{B}\}=\mu_{*}(E)=$ $\sup \{\mu(A): E \supset A \in \mathscr{B}\}$. One sets, for $E \in \hat{\mathscr{B}}^{\mu}, \mu(E)=\mu_{*}(E)=\mu^{*}(E)$. $\hat{\eta}_{\mu}$ is then the ideal of $\mu$ negligible sets in $\hat{\mathscr{B}}^{\mu}$ and $\hat{\mathscr{B}} \Delta \hat{\eta}_{\mu} \subset \mathscr{\mathscr { B }}^{\mu}$. One may ask whether $\hat{\mathscr{B}}^{\mu}$ is ever $d_{\mu}$-complete. To answer this it is convenient to characterize $\widehat{\mathscr{B}}^{\mu}$ in terms of the Stone space $X_{\mathscr{B}}$.

Let $j_{\mathscr{B}}(x)=\{A \in \mathscr{B}, x \in A\} \in X_{\mathscr{B}}$. The mapping $j_{\mathscr{B}}$ from $X$ to $X_{\mathscr{B}}$ is such that if $A$ is in $\mathscr{B}$ then $[A]=\overline{j_{\mathscr{B}}(A)}$ so that $A=j_{\mathscr{O}^{\prime}}^{-1}([A])$. The inverse image of the clopen algebra of $X_{\mathscr{B}}$ is the algebra $\mathscr{B}$. It is convenient to identify $X$ with the dense subset $j_{\mathscr{B}}(X)$ of $X_{\mathscr{O}}$ even though this is only proper if $j_{\mathscr{B}}$ is injective iff $\mathscr{B}$ separates $X$.

Proposition 6. $E \subset X$ is in $\hat{\mathscr{B}}^{\mu}$ iff there is a closed $G_{\dot{j}}, F$ and an open $F_{o}, G$, in $X_{\mathscr{B}}$ with $G \subset F, \tilde{\mu}(F \backslash G)=0$, and $j_{\mathscr{B}}^{-1}(G) \subset E \subset$ $j_{\mathscr{\mathscr { S }}}^{-1}(F)$. In particular $\tilde{\mu}\left(\overline{\partial j_{\mathscr{B}}(E)}\right)=0$.

Proof. Let $E \in \hat{\mathscr{B}}^{\mu}$. Let $\left\{A_{n}\right\}$ be an increasing sequence in $\mathscr{B}$ and $\left\{A^{n}\right\}$ be a decreasing sequence in $\mathscr{B}$ with $A_{n} \subset E \subset A^{n}$ so that $\mu\left(A^{n} \backslash A^{n}\right) \rightarrow 0$ as $n \rightarrow \infty$. Let $G=\bigcup_{n=1}^{\infty}\left[A_{n}\right]$ and $F=\bigcap_{n=1}^{\infty}\left[A^{n}\right]$. We have $G \subset F$ with $\tilde{\mu}(F \backslash G)=0$ and we have $j_{\mathscr{B}}^{-1}(G)=\bigcup_{n=1}^{\infty} A_{n} \subset E \subset$ $\bigcap_{n=1}^{\infty} A^{n}=j_{\mathscr{S}}^{-1}(F)$.

Conversely, if $G$ is an open $F_{\sigma}$ and $F$ a closed $G_{\delta}$ in $X_{\mathscr{G}}$ with $j_{\mathscr{S}}^{-1}(G) \subset E \subset j_{\mathscr{S}^{\prime}}^{-1}(F)$ and with $\tilde{\mu}(F \backslash G)=0$ then $G=\bigcup_{n=1}^{\infty}\left[A_{n}\right]$ and $F=$ $\bigcap_{n=1}^{\infty}\left[A^{n}\right]$ with $\left\{A^{n}, A_{n}: n \in N\right\} \subset \mathscr{B}$ with $A_{n} \subset E \subset A^{n}$ for $n \in N$ and with $\mu\left(A^{n} \backslash A_{n}\right) \rightarrow 0$ as $n \rightarrow \infty$. Thus, $E \in \widehat{\mathscr{B}}^{n}$.

Proposition 7. $\widehat{\mathscr{B}}^{\mu}$ is $d_{\mu^{-}}$complete iff (i) $\hat{\mathscr{B}}^{\mu}$ is a $\sigma$-algebra of subsets of $X$ and (ii) $\mu$ is countably additive on $\hat{\mathscr{B}}^{\mu}$. In this case $\hat{\mathscr{B}}^{\prime \prime}$ is $\mu$-complete as a $\sigma$-algebra. 
Proof. From Proposition 2, $d_{\mu}$-completeness of $\hat{\mathscr{B}}^{\mu}$ follows from (i) and (ii). Also, $d_{\mu}$-completeness of $\hat{\mathscr{B}}^{\mu}$ implies (ii) and that $\hat{\mathscr{B}}^{\mu}$ is $\sigma$-complete as a Boolean algebra. If $\left\{E_{n}\right\}$ is an increasing sequence in $\widehat{\mathscr{B}}^{\mu}$ we must show that $E=\bigcup_{n=1}^{\infty} E_{n} \in \hat{\mathscr{B}}^{\mu}$. Let $E^{\infty}$ be the supremum of $\left\{E_{n}\right\}$ in $\hat{\mathscr{B}}^{\mu}$. Let $\left\{A_{n}\right\}$ be chosen increasing in $\mathscr{B}$ with $A_{n} \subset E_{n}$ and $\mu\left(E_{n} \backslash A_{n}\right)<1 / n$ for all $n$. Let $\left\{A^{n}\right\}$ be chosen decreasing in $\mathscr{B}$ with $E^{\infty} \subset A^{n}$ and $\mu\left(A^{n} \backslash E_{\infty}\right)<1 / n$ for all $n$. We have $A_{n} \subset$ $E \subset A^{n}$ and $\mu\left(A^{n} \backslash A_{n}\right) \rightarrow 0$ as $n \rightarrow \infty$. Thus, $E \in \hat{\mathscr{B}}^{\mu}$.

Proposition 8. $\hat{\mathscr{B}}^{\mu}$ is $d_{\mu}$-complete iff $\tilde{\mu}$ is a category measure on $X_{\mathscr{O} \mu}$.

Proof. A residual Radon measure is a category measure on its support, [2].

Let $\hat{\mathscr{B}}^{\mu}$ be $d_{\mu}$-complete. We must show that if $\Theta$ is an open set in $X_{\mathscr{O}_{\mu}}$ then $\widetilde{\mu}(\partial \Theta)=0$, [3]. There is an open $F_{\sigma} \Theta^{\prime} \subset \Theta$ with $\tilde{\mu}\left(\Theta / \Theta^{\prime}\right)=0$. Let $\widetilde{\Theta}^{\prime}$ be an open $F_{\sigma}$ in $X_{\mathscr{B}}$ with $\widetilde{\Theta}^{\prime} \cap X_{\mathscr{A}_{\mu}}=\Theta^{\prime}$ (where $X_{\mathscr{\sigma _ { \mu } \mu}}$ is considered to be $\left.\operatorname{supp}(\tilde{\mu}) \subset X_{\mathscr{O}}\right)$. We have $\tilde{\mu}\left(\partial \tilde{\theta}^{\prime}\right)=0$. Thus, considering closure in $X_{\mathscr{B}_{\mu}}, \tilde{\mu}\left(\partial \theta^{\prime}\right)=0$. Since $X_{\mathscr{S}_{\mu}}=\operatorname{supp}(\tilde{\mu}), \bar{\theta}^{\prime}=\bar{\theta}$. Since $\Theta$ differs from $\Theta^{\prime}$ by a $\tilde{\mu}$-negligible set and $\Theta^{\prime}$ differs from $\bar{\Theta}$ by a negligible set $\tilde{\mu}(\partial \Theta)=0$ which shows that $\tilde{\mu}$ is residual on $X_{\mathscr{B}_{\mu}}$.

Let $\tilde{\mu}$ be residual on $X_{\mathscr{S}_{\mu}}$. From Oxtoby [20, Theorem 4] any Borel set $A$ in $X_{\mathscr{B}_{\mu}}$ has the property that $\tilde{\mu}(A)=\tilde{\mu}\left(A^{0}\right)=\tilde{\mu}(\bar{A})$. Thus, if $A$ is a Baire set in $X_{\mathscr{\sigma} \mu}$ there is an open $F_{\sigma} G \subset A$ and a closed $G_{\delta} F \supset A$ with $\tilde{\mu}(F \backslash G)=0$. Represent $G$ as $\bigcup_{n=1}^{\infty}\left\{\left[A_{n}\right] \cap X_{\mathscr{O}_{\mu}}\right\}$ where $\left\{A_{n}\right\} \subset \mathscr{B}$ is increasing, and $F$ as $\bigcap_{n=1}^{\infty}\left\{\left[A^{n}\right] \cap X_{\mathscr{B}}\right\}$ with $\left\{A^{n}\right\} \subset$ $\mathscr{B}$ decreasing with $A_{n} \subset A^{n}$ for all $n$, and with $\mu\left(A^{n} \backslash A_{n}\right)=0$. Let $E \subset X$ be $\bigcap_{n=1}^{\infty} A^{n}$. Since $A_{n} \subset E \subset A^{n}$ for all $n$ we have $E \in \hat{\mathscr{B}}^{\mu}$. It is easily checked that $E$ is the $d_{\mu}$-limit of the Cauchy sequence $\left\{A_{n}\right\} \subset \mathscr{B}$ and that $E$ corresponds to the element $A$ in the $d_{\mu}$-completion of $\mathscr{B}_{\mu}$ as given in Proposition 5.

By Proposition $4, \hat{\mathscr{B}}^{\mu}$ is $d_{\mu}$-complete iff every $\nu$ with $|\nu|=\mu$ has a $\hat{\mathscr{B}}^{\mu}$-measurable Radon-Nikodym derivative. One may ask what is the case if one allows Eudoxus integrable, [14], Radon-Nikodym derivatives. A bounded function $f$ is Eudoxus integrable iff there an increasing sequence $\left\{f_{n}\right\}$ of bounded $\mathscr{B}$-measurable functions and a decreasing sequence $\left\{f^{n}\right\}$ of bounded $\mathscr{B}$-measurable functions such that $f_{n} \leqq f \leqq f^{n}$ for all $n$ and $\lim _{n \rightarrow \infty} \int f^{n}-f_{n} d_{\mu}=0$. Since bounded $\widehat{\mathscr{B}}^{\mu}$-measurable functions are Eudoxus integrable no more Endoxus integrable functions are obtained if one only requires $\hat{\mathscr{B}}^{\mu}$-measurability of $\left\{f_{n}\right\}$ and $\left\{f^{n}\right\} . \int f d_{\mu}$ is defined by $\lim _{n \rightarrow \infty} \int f_{n} d_{\mu}$ or $\lim _{n \rightarrow \infty} \int f^{n} d_{\mu}$. 
COROLLARY 8.1. $\widehat{\mathscr{B}}^{\mu}$ is $d_{\mu}$-complete iff every $\nu$ with $|\nu|=\mu$ has a Eudoxus integrable Radon-Nikodym derivative.

Proof. One direction is clear. For the other suppose that all $\nu$ with $|\nu|=\mu$ have Eudoxus integrable derivatives. We shall consider $X$ as identified with a subset of $X_{\mathscr{G}}$ via the map $j_{\mathscr{B}}$. Let $\nu$ have $|\nu|=\mu$ and let $f$ be a Eudoxus integrable Radon-Nikodym derivative. Let $\left\{f_{n}\right\}$ and $\left\{f^{n}\right\}$ be the monotone sequences of bounded $\mathscr{B}$-measurable functions with $f_{n} \leqq f \leqq f^{n}$ for all $n$ so that $\lim _{n \rightarrow \infty} \int f^{n}-f_{n} d_{\mu}=0$. Let $\left\{\tilde{f}_{n}\right\}$ and $\left\{\widetilde{f}^{n}\right\}$ be the corresponding sequences in $\mathscr{C}\left(X_{\mathscr{B}}\right)$. Let $\breve{f}=\inf _{n} \widetilde{f}^{n}$ and $\hat{f}=\sup _{n} \tilde{f}_{n}$. $\check{f}$ is upper semicontinuous and $\check{f}$ is lower semi-continuous. The restrictions of $\check{f}$ and $\hat{f}$ to $X$ are themselves Eudoxus integrable Radon-Nikodym derivatives of $\nu$. Both $|\hat{f}|$ and $|\check{f}|$ are equal to $1 \tilde{\mu}$ a.e. Let $K$ be the compact $G_{i}\{\check{f} \geqq 1\}$. One has $\check{f}=\chi_{K}-\chi_{K^{c}} \tilde{\mu}$ a.e. Since $\nu$ was arbitrary $\tilde{\nu}$ could have been of the form $\left(\chi_{\theta}-\chi_{\theta c}\right) \tilde{\mu}$ for an open set $\theta$ in $X_{\mathscr{O}}$. Thus, for each open $\theta$ there is a compact $G_{\delta} K$ in $X_{\mathscr{A}}$ with $\tilde{\mu}(\theta \Delta K)=0$. The closure of $\theta \cap X_{\mathscr{\diamond}_{\mu}}$ must be contained in $K \cap X_{\mathscr{B}_{\mu}}$ since $\operatorname{supp}(\tilde{\mu})=X_{\mathscr{A} \mu}$. Thus, in $X_{\mathscr{O}_{\mu}}, \tilde{\mu}\left(\partial\left(\theta \cap X_{\mathscr{O}_{\mu}}\right)\right)=0$. Since

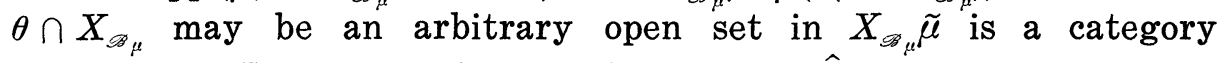
measure on $X_{\mathscr{B} \mu}$. Proposition 8 shows that $\hat{\mathscr{B}}^{\mu}$ is $d_{\mu}$-complete.

REMaRKs. Can Eudoxus integrability be replaced by $\mu$-integrability? Recall that $f$ is $\mu$-integrable iff there is a sequence of simple $\mathscr{B}$-measurable functions which converges to $f$ in $\mu$-measure or in $\mu$-probability.

The maximal ideal space $Z_{\tilde{\mu}}$ of $L^{\infty}\left(X_{\mathscr{乃}}, \tilde{\mu}\right)$ is the Gleason space or projective cover of $X_{\mathscr{C}_{\mu}}$ iff $\tilde{\mu}$ is a category measure on $X_{\mathscr{}}$, [3]. This is true iff the projection dual to the injection $C\left(X_{\mathscr{B} \mu}\right) \subset L^{\infty}\left(X_{\mathscr{B} \mu}\right.$, $\tilde{\mu})$ is irreducible. This yields a method for constructing $\hat{\mathscr{B}}^{\mu}$ which are $d_{\mu}$-complete, yet such that $\mathscr{B} \Delta \hat{\eta}_{\mu}$ isn't $d_{\mu}$-complete no matter how $\mathscr{B}$ is represented as an algebra of sets. One need only take an irreducible totally disconnected image $Y$ of the maximal ideal space $Z$ of $L^{\infty}(\Omega, \Sigma, P)$ where $(\Omega, \Sigma, P)$ is a probability measure space. Letting $\mathscr{B}$ be the clopen algebra of $Y$ one has $Y=X_{\mathscr{B}}$. One may take $X\left(=j_{\mathscr{B}}(X)\right)$ any dense subset of $X_{\mathscr{B}}$ regarding $\mathscr{B}$ now to be equal to it's trace on $X$. One way to obtain $Y$ from $Z$ is to identify two nonisolated points in $Z$ (or even to identify a closed nowhere dense subset of $Z$ ).

CoRollary 8.2. There exists a set $X$, a Boolean algebra $\mathscr{B}$ of subsets of $X$ and a strictly positive finitely additive probability $\mu$ on $\mathscr{B}$ so that $\mathscr{B}_{\mu}$ isn't $d_{\mu}$-complete yet $\left(\mathscr{\mathscr { B }}^{\mu}\right)_{\mu}$ is $d_{\mu^{-}}$-complete. 
The completion $\mathscr{B}_{\tilde{\mu}}^{0}$ of $\mathscr{B}_{\mu}$ under $d_{\tilde{\mu}}$ is a complete metrizable abelian topological group when the group operation is symmetric difference. Since $\mathscr{B}_{\mu}$ is a dense subgroup of $\mathscr{B}_{\tilde{\mu}}^{0}$ the regular open algebra of $\mathscr{B}_{\mu}$ is isomorphic to that of $\mathscr{B}_{\tilde{\mu}}^{0}$, [18], [20]. If $F$ is a closed subset of $\mathscr{B}_{\mu}$ its interior is the intersection of $\bar{F}^{0}$ with $\mathscr{B}_{\mu}$ where closure and interior are taken in $\mathscr{B}_{\tilde{\mu}}^{0}$. Thus, $F$ is nowhere dense in $\mathscr{B}_{\mu}$ iff $F$ is nowhere dense in $\mathscr{B}_{\tilde{\mu}}^{0}$. Thus, $\mathscr{B}_{\mu}$ is meager in itself iff it is meager in $\mathscr{B}_{\tilde{\mu}}^{0}$. When $\mathscr{B}_{\mu}$ is incomplete yet nonmeager it must be badly behaved as a subset of $\mathscr{B}_{\tilde{\mu}}^{0}$. In Kelley [16, Problem 6P] it is shown that any nonmeager dense subgroup of a Baire topological group fails to have the property of Baire.

Proposition 9. If $\mathscr{B}_{\mu}$ is not complete then it either

(a) is meager in itself under $d_{\mu}$ or

(b) fails to have the property of Baire in its $d_{\mu}$-completion.

When $\mathscr{B}_{\mu}$ is $d_{\mu}$-incomplete it may be meager. One instance is when $\mathscr{B}_{\mu}$ is countably infinite in particular when $\mathscr{B}$ is countable and $\mathscr{B}_{\mu}$ is infinite. In this case each point of $\mathscr{B}_{\mu}$ is nowhere dense hence $\mathscr{B}$ is meager. In quite a few instances $\mathscr{B}_{\mu}$ will be meager.

Proposition 10. Let $\mu \in B A_{1}^{+}(\mathscr{B})$. If $A \in \mathscr{B}_{\mu}$ (or $\left.\mathscr{B}\right)$ let $\mathscr{F}(A)=$ $\left\{A^{\prime} \in \mathscr{B}_{\mu}: A^{\prime} \subset A\right\}$ and let $\mathscr{F}(A)=\left\{A^{\prime} \in \mathscr{B}_{\mu}: A \subset A^{\prime}\right\}$ be the principal ideal and filter in $\mathscr{B}_{\mu}$ generated by $A$.

(a) Both $\mathscr{F}(A)$ and $\mathscr{I}(A)$ are $d_{\mu}$-closed.

(b) $\mathscr{I}(A)$ is nowhere dense iff $A^{\mathrm{c}}$ isn't a finite union of $\mu$ atoms and is open if $A^{c}$ is a finite union of r-atoms.

(c) $\mathscr{F}(A)$ is nowhere dense iff $A$ isn't a finite union of r-atoms and is open if $A$ is a finite union of $\mu$-atoms.

Proof. Only statements about $\mathscr{I}(A)$ need be proven for the statements about $\mathscr{F}(A)$ follow from those for $\mathscr{J}(A)$ upon applying the isometry $E \rightarrow E^{c}$.

(a) To show that $\mathscr{F}(A)$ is $d_{\mu}$-closed consider a sequence $\left\{A_{n}\right\} \subset$ $\mathscr{J}(A)$ converging to $C \in \mathscr{B}_{\mu}$. We have $\mu\left(C \backslash A_{n}\right)=\mu(C \backslash A)+\mu(C \cap$ $\left.\left(A \backslash A_{n}\right)\right) \geqq \mu(C \backslash A)$. From $\lim _{n \rightarrow \infty} \mu\left(C \backslash A_{n}\right)=0$ it follows that $\mu(C \backslash A)=0$ so $C \in \mathscr{J}(A)$. This establishes (a).

(b) If $A^{c}$ is a finite union of atoms then $\mathscr{B}_{\mu}=\cup\{\mathscr{I}(A) \Delta F$ : $\left.F \subset A^{c}\right\}$, where $\mathscr{J}(A) \Delta F=\{E \Delta F: E \in \mathscr{J}(A)\}$, is a finite disjoint union. The map $E \rightarrow E \Delta F$ is an isometry of $\mathscr{B}_{\mu}$ for $d_{\mu}$. Thus, $\mathscr{I}(A) \Delta F$ is a closed set for each $F \subset A^{c}$. Since $\mathscr{B}_{\mu}$ is a finite union of disjoint closed sets each is a clopen set. Thus, $\mathscr{J}(A)$ is clopen.

Conversely, if $A^{c}$ is not a finite union of atoms there are $F \subset \mathscr{B}_{\mu}$ $F \subset A^{c}$ with $\mu(F)>0$ but arbitrarily small. If $A^{\prime} \in \mathscr{I}(A)$ then 
$d_{\mu}\left(A^{\prime}, A^{\prime} \cup F\right)$ is arbitrarily small yet $A^{\prime} \cup F \notin \mathscr{I}(A)$. Thus, no $A^{\prime} \in \mathscr{I}(A)$ is an interior point of $\mathscr{I}(A)$. Thus, $\mathscr{J}(A)$ is nowhere dense.

To show that $\mathscr{B} \mu$ was meager it would suffice to show that there was a countable family $\left\{A_{n}\right\} \subset \mathscr{B}_{\mu} \backslash\{\phi\}$, with $\mathscr{F}\left(A_{n}\right)$ nowhere dense for all $n$, with $\mathscr{B}_{\mu}=\bigcup_{n=1}^{\infty} \mathscr{F}\left(A_{n}\right)$. That is, $\left\{A_{n}\right\}$ should be a family such that if $A \in \mathscr{B}_{\mu}$ there is an $A_{n}$ with $A_{n} \subset A$ and so that no $A_{n}$ is a finite union of atoms. A collection $\left\{A_{\alpha}\right\} \subset \mathscr{B}_{\mu} \backslash\{\phi\}$ such that any $A \in \mathscr{B} \mathscr{B}_{\mu} \backslash\{\phi\}$ contains an $A_{\alpha}$ is called a pseudo base of the algebra $\mathscr{B}_{\mu}$, [21]. Included in any pseudo base for $\mathscr{B}_{\mu}$ is the, at most countable, collection of atoms. If every $A \in \mathscr{B}_{\mu}$ contains an atom then the collection of atoms is a pseudo base and is minimal as a pseudo base. This is the case iff $X_{\mathscr{B}_{\mu}}$ is the closure of its countable set of isolated points iff $X_{\mathscr{B}_{\mu}}$ is between $N \cup\{\infty\}$ and $\beta N$ as a compact Hausdorff space.

Proposition 11. Suppose that $\mathscr{B}_{\mu}$ is such that there exists an $A \in \mathscr{B}_{\mu} \backslash\{\phi\}$ not containing a $\mu$-atom and such that the restriction of $\mathscr{B}_{\mu}$ to $A$ has a countable pseudo base. $\mathscr{B}_{\mu}$ is meager.

Proof. Let $\mu_{A}$ be the restriction of $\mu$ to $A$ normalized to be a probability measure. $\mathscr{B}_{\mu_{A}}$ is the restriction of $\mathscr{B}_{\mu}$ to $A$. $\mathscr{B}_{\mu}$ is meager as the preceding remarks have shown. Let $\mu_{A^{c}}$ be the normalized restriction of $\mu$ to $A^{c}$. If $\mu_{A^{c}}$ doesn't exist then $\mathscr{B}_{\mu}=$ $\mathscr{B}_{\mu_{A}}$ is meager. It is easily verified that $\mathscr{B}_{\mu}$ may be represented as the product $\mathscr{B}_{\mu_{A}} \times \mathscr{B}_{\mu_{A}}$. Furthermore the metric $d_{\mu}$ is given by $d_{\mu}\left(\left(E_{1}, F_{1}\right), \quad\left(E_{2}, F_{2}\right)\right)=\mu(A) d_{\mu_{A}}\left(E_{1}, E_{2}\right)+\mu\left(A^{c}\right) d_{\mu_{A^{c}}}\left(F_{1}, F_{2}\right) \quad$ which yields a topology on $\mathscr{B}_{\mu_{A}} \times \mathscr{B}_{\mu_{A^{c}}}$ which is the product topology. Since $\mathscr{B}_{\mu_{A}}$ is meager so is $\mathscr{B}_{\mu_{A}} \times \mathscr{B}_{\mu_{A}}=\mathscr{B}_{\mu}$.

REMARK. Every nonnegligible element of $\hat{\mathscr{B}}^{\mu}$ contains a nonnegligible element of $\mathscr{B}$ hence this proposition extends to the case of $\hat{\mathscr{B}}^{\mu}$. We may even extend this proposition to cover the case of the Boolean algebra completion of $\mathscr{B}$ or $\hat{\mathscr{B}}^{\mu}$.

Proposition 12. If $\mathscr{B}$ is an infinite Boolean algebra there is a probability measure $\mu$ on $\mathscr{B}$ such that $\mathscr{B}_{\mu}$ is meager, $\mu$ may be taken to be non-atomic if $\mathscr{B}$ admits a non-atomic measure and may always be chosen to be atomic otherwise.

Proof. If $\mathscr{B}$ admits a non-atomic measure $\mu$ there is, [4], [24] a countable subalgebra $\mathscr{B}_{0}$ of $\mathscr{B}$ isomorphic to the clopen algebra 
of the Cantor set $\Delta$. The algebra $\mathscr{B}_{0}$ has a countable base hence a countable pseudo base. Let $\Phi: X_{\mathscr{G}} \rightarrow X_{\mathscr{F}_{0}} \cong \Delta$ be the canonical surjection. Let $\tilde{\nu}$ be any non-atomic Radon probability measure on $X_{\mathscr{B}_{0}}$ with support equal to $X_{\mathscr{B}_{0}}$. Let $X$ be a minimal closed subset of $X_{\mathscr{A}}$ such that $\Phi(Y)=X_{\mathscr{B}_{0}}$. The map $\Phi$ is irreducible on $Y$, [27], [4], hence $Y$ has a countable pseudo base, [27]. Let $\tilde{\mu}$ be a Radon probability measure on $Y$ (hence on $X_{\mathscr{G}}$ ) whose image under $\Phi$ is $\tilde{\nu}$. As in [4], $\tilde{\mu}$ is non-atomic on $X_{\mathscr{B}}$. Let $\mu$ be the measure on $\mathscr{B}$ corresponding to $\tilde{\mu}$ under the Stone correspondence. We have $Y=$ $X_{\mathscr{B} \mu}$. Since $Y$ has a countable pseudo base and $\mu$ is non-atomic it follows from Proposition 11 that $\mathscr{B}_{\mu}$ is meager.

If $\mathscr{B}$ admits no nonzero non-atomic measure there is no nonzero non-atomic Radon measure on $X_{\mathscr{B}}$ hence $X_{\mathscr{B}}$ is scattered, [27], as is any closed subset. Since $X_{\mathscr{B}}$ is infinite there is a probability $\tilde{\mu}=\sum_{n=1}^{\infty} 2^{-n} \delta_{x_{n}}$ where $\left\{x_{n}\right\}$ is an infinite sequence in $X_{\mathscr{B}}$. The support $Y$ of $\tilde{\mu}$ is a separable scattered space. If $\mu$ is the measure on $\mathscr{B}$ corresponding to $\tilde{\mu}$ under the Stone correspondence then $Y=X_{\mathscr{\beta} \mu}$. The algebra $\mathscr{B}_{\mu}$ is the clopen algebra of $Y$. Every clopen set in $Y$ contains one of the countable many isolated points. Thus, $\mathscr{B}_{\mu}$ has a countable pseudobase.

REMARK. Again if $\mathscr{B}$ is an algebra of sets this proposition is valid for $\hat{\mathscr{B}}^{\mu}$.

We may improve Proposition 11 to some extent in the following proposition.

Proposition 13. Let $\mathscr{B}$ be an algebra and $\mu$ be a finitely additive probability on $\mathscr{B}$ so that $\mathscr{B}_{\mu}$ has a nonprincipal ultrafilter with a countable base. $\mathscr{B}_{\mu}$ is $d_{\mu}$-meager.

Proof. Let $\left\{A_{n}: n \in N\right\}$ be a countable base for an ultrafilter $\mathscr{F}$ in $\mathscr{B}_{\mu}$ so that $A_{n} \supset A_{n+1}$ for all $n$ and so that $\mu\left(A_{n} \backslash A_{n+1}\right)>0$ for all $n$. $\mathscr{F}$ is equal to $\bigcup_{n=1}^{\infty} \mathscr{F}\left(A_{n}\right)$. By Proposition 10 each $\mathscr{F}\left(A_{n}\right)$ is nowhere dense hence $\mathscr{F}$ is meager for $d_{\mu}$. Consequently, the maximal ideal $\mathscr{F}$ dual to $\mathscr{F}$ is also meager. Since $\mathscr{B}_{\mu}=\mathscr{F} \cup$ $\mathscr{I} . \mathscr{B}_{\mu}$ is meager.

Proposition 14. For any infinite cardinal number $m$ there is a Boolean algebra $\mathscr{B}$ and a finitely additive probability $\mu$ on $\mathscr{B}$ so that $\mathscr{B}_{\mu}$ is meager and has density character $m$.

Proof. (The density character of a topological space is the minimum cardinal number of a dense subset.) 
Let $\mathscr{B}^{\prime}$ be the clopen algebra of the maximal ideal space $X_{\mathscr{G}^{\prime}}$ of $L^{\infty}\left(\{0,1\}^{m}, \hat{\mu}\right)$ where $\hat{\mu}$ is the coin flip measure. Let $\tilde{\mu}$ be the probability Radon measure on $X_{\mathscr{B}^{\prime}}$ corresponding to $\hat{\mu}$ under the Banach lattice isomorphism between $\mathscr{C}\left(X_{\mathscr{B}^{\prime}}\right)$ and $L^{\infty *}\left(\{0,1\}^{m}, \hat{\mu}\right)$ dual to that between $\mathscr{C}\left(X_{\mathscr{O}^{\prime}}\right)$ and $L^{\infty}\left(\{0,1\}^{m}, \hat{\mu}\right)$. Let $\mu$ be the countably additive probability on $\mathscr{B}^{\prime}$ corresponding to $\tilde{\mu}$ under the Stone correspondence. Consider the cardinal $m$ to be the first ordinal of cardinal $m$. Let $\hat{A}_{\alpha}$, for $\alpha$ an ordinal less than $m$, denote the clopen subset of $\{0,1\}^{m}$ consisting of those elements whose $\alpha$ th coordinate is 0 . Let $A_{\alpha}$ be the element of $\mathscr{B}^{\prime}$ corresponding to $\hat{A}_{\alpha}$ for ordinals $\alpha<m$. The subalgebra of $\mathscr{B}^{\prime}$ generated by $\left\{A_{\alpha}: \alpha<m\right\}$ is of cardinality $m$ and is $d_{\mu}$-dense in $\mathscr{B}^{\prime}$. Thus, the $d_{\mu}$ density character of $\mathscr{B}^{\prime}$ is at most $m$. It is easily verified that $d_{\mu}\left(A_{\alpha}, A_{\beta}\right)=$ $1 / 2$ for all $\alpha \neq \beta$. Thus, the density character of $\mathscr{B}^{\prime}$ is at least $m$. This establishes the (well known) fact that $\mathscr{B}^{\prime}$ has density character $m$. The same reasoning shows that $\mathscr{I}\left(A_{\alpha}^{c}\right)$, the principal ideal in $\mathscr{B}^{\prime}$ generated by $A_{\alpha}^{c}$ has density character $m$ as a closed subset of $\mathscr{B}^{\prime}$. Choose a decreasing sequence $\left\{E_{n}: n \in N\right\} \subset \mathscr{B}^{\prime}$ with $E_{1}=A_{1}$ and $\left.\mu\left(E_{j}\right\rangle E_{j+1}\right)>0$. Let $\mathscr{F}$ be the filter $\bigcup_{n=1}^{\infty} \mathscr{F}\left(E_{n}\right)$ and let $\mathscr{J}$ be the ideal dual to $\mathscr{F}$. Let $\mathscr{B}$ be the algebra $\mathscr{F} \cup \mathscr{F}$. From Proposition 13, $\mathscr{B}=\mathscr{B}_{\mu}$ is $d_{\mu}$-meager. Since $\mathscr{I}\left(A_{1}^{c}\right) \subset \mathscr{I}$ there is a closed subset of the metric space $\mathscr{B}$ of density character $m$. Thus, $\mathscr{B}$ has density character at least $m$ and, since $\mathscr{B} \subset \mathscr{B}^{\prime}$, the density character of $\mathscr{B}$ is equal to $m$.

REMARK. Under this construction $\mu$ is never countably additive. Can $\mu$ be constructed to be countably additive?

If one wishes to find an algebra $\mathscr{B}$ and a finitely additive probability measure $\mu$ on $\mathscr{B}$ so that $\mathscr{B}_{\mu}$ is not meager for $d_{\mu}$ yet not complete one should choose $\mathscr{B}_{\mu}$ very large in its $d_{\mu}$-completion $\mathscr{B}_{\tilde{\mu}}^{0}$. Considering $\mathscr{B}_{\mu}$ as a subalgebra of $\mathscr{B}_{\tilde{\mu}}^{0}$ one has the Stone space

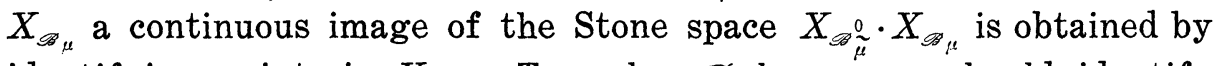
identifying points in $X_{\mathscr{A}_{\tilde{\mu}}^{0}}$. To make $\mathscr{B}_{\mu}$ large one should identify as few points as possible. For our construction we will start out with a given infinite hyperstonian space $Z$ satisfying the countable chain condition so that $Z$ is the maximal ideal space of $L^{\infty}(\Omega, \Sigma, P)$ for some probability measure space $(\Omega, \Sigma, P)$ not consisting of finitely many $P$ atoms. We will consider $\tilde{\mu}$ to be the Radon probability measure on $Z$ associated with $P$ and will denote by $\mathscr{B}_{\tilde{\mu}}^{0}$ the clopen algebra of $Z$ so that $Z=X_{\mathscr{P}_{\tilde{\mu}}^{0}}$. We will identify finitely many nonisolated points of $Z$ to obtain a totally disconnected $Z^{\prime}$ whose clopen algebra will be denoted by $\mathscr{B}$. We will again denote by $\tilde{\mu}$ the Radon probability measure on $Z^{\prime}$ which is the image of $\tilde{\mu}$ under the canonical projection of $Z$ onto $Z^{\prime}$. By $\mu$ we will mean the 
finitely additive probability on $\mathscr{B}$ (or $\mathscr{B}_{\tilde{\mu}}^{0}$ ) corresponding to $\tilde{\mu}$. Since $\mu$ is strictly positive on $\mathscr{B}_{\tilde{\mu}}^{0}$ and on $\mathscr{B}=\mathscr{B}_{\mu}$ and $Z^{\prime}=X_{\mathscr{B}}$. Consequently, we are in the desired setting for this proposition.

Proposition 15. Let $(\Omega, \Sigma, P)$ be a (countably additive) probability measure space not consisting of finitely many $P$-atoms. There is a subalgebra $\widetilde{\Sigma}$ of $\Sigma$ so that $\widetilde{\Sigma}_{P}$ is incomplete, nonmeager for $d_{P}$ with $d_{P}$-completion $\Sigma_{P}$.

Proof. Assume the notation in the paragraph preceding this proposition. If we show that $\mathscr{B}_{\mu}$ is $d_{\mu}$-incomplete we may obtain $\widetilde{\Sigma}$ from $\mathscr{B}_{\mu} \subset \mathscr{B}_{\widetilde{\mu}}^{0}=\Sigma_{P}$ by using a lifting $\lambda$ for $L^{\infty}(\Omega, \Sigma, P)$ and taking $\widetilde{\Sigma}$ to be the image of $\mathscr{B}_{\mu}$ under $\lambda$.

Let $\left\{x_{1}, \cdots, x_{n}\right\}$ be the points identified in $Z$ to get $x \in Z^{\prime}$. Each of $\left\{x_{1}, \cdots, x_{n}\right\}$ is an ultrafilter on $\mathscr{B}_{\tilde{\mu}}^{0}$ which contains elements of $\mathscr{B}_{\tilde{\mu}}^{0}$ of arbitrarily small $\mu$ measure (since each $x_{i}$ is nonisolated). Let $\mathscr{F}$ be the filter $x_{1} \cap \cdots \cap x_{n}$ which again contains elements of arbitrarily small $\tilde{\mu}$ measure. Let $\mathscr{J}$ be the ideal of $\mathscr{B}_{\tilde{\mu}}^{0}$ dual to $\mathscr{F}$ so $\mathscr{F}=\left\{A^{c}: A \in F\right\}$. $\mathscr{F}$ is a subgroup of $\mathscr{B}_{\tilde{\mu}}^{0}$ and is dense for $d_{\mu}$ since $\mathscr{F}$ contains sets of arbitrarily small measure. Thus, $\mathscr{I}$ is either meager or fails to have the property of Baire. $\mathscr{J}$ is a subgroup of $\mathscr{B}_{\tilde{\mu}}^{0}$ of finite index. This is because $\mathscr{J}=\mathscr{I}_{1} \cap \cdots \cap \mathscr{F}_{n}$ where $\mathscr{J}_{j}$ is the maximal ideal of $\mathscr{B}_{\tilde{\mu}}^{0}$ dual to the ultrafilter $x_{j}$. No subgroup of $\mathscr{B}_{\tilde{\mu}}^{0}$ of finite index can be meager. Thus, $\mathscr{J}$ is non meager. The algebra $\mathscr{B}_{\mu}$ is easily seen to be $\mathscr{I} \cup \mathscr{F}$ hence is a nonmeager, dense, incomplete subgroup of $\mathscr{B}_{\tilde{\mu}}^{0}$. Thus, $\mathscr{B}_{\mu}$ fails to have the property of Baire.

REMARKs. (1) It may be shown that as constructed, $P$ is not countably additive on $\widetilde{\Sigma}$ nor is $\widetilde{\Sigma}$ complete as a Boolean algebra. Is it true that if the projection of $X_{\tilde{\mathscr{A}}_{\mu}}$ onto $X_{\mathscr{C}_{\mu}}$ is irreducible that $\mathscr{B}_{\mu}$ is nonmeager? We conclude with a variation of Proposition 14 valid for complete Boolean algebras but with density characters restricted to cardinals between $\boldsymbol{\aleph}_{0}$ and $2^{\boldsymbol{N}_{0}}$.

Proposition 16. Let $\mathscr{B}$ be an infinite complete Boolean algebra and $m$ a cardinal number between $\boldsymbol{\aleph}_{0}$ and $2^{\aleph_{0}}$. There is a finitely additive probability measure $\mu$ on $\mathscr{B}$ such that $\mathscr{B}_{\mu}$ is $d_{\mu}$-meager and has density character $m$.

Proof The first step of the proof is the construction of a probability measure $\mu_{1}$ on $2^{N}$ so that $2^{N}$ has $d_{\mu_{1}}$ density character $m$. Let $\mathscr{A}_{0}$ be a free subalgebra of $2^{N}$ with $m$ generators (since $m \leqq 2^{\aleph_{0}}$ $\mathscr{A}_{0}$ exists). On $\mathscr{A}_{0}$ let $\mu_{1}$ be the usual coin toss measure so that each 
of the $m$ generators of $\mathscr{A}_{0}$ receives measure $1 / 2$ and so that the generators are $\mu_{1}$-independent. The density character of $\mathscr{A}_{0}$ for $d_{\mu_{1}}$ is equal to $m$. Under any extension of $\mu_{1}$ to $2^{N}, 2^{N}$ will have $d_{\mu_{1}}$-density character at least $m$. If $\mu_{1}$ is extended to $2^{N}$ so that $\mathscr{A}_{0}$ is $d_{\mu_{1}}$-dense in $2^{N}$ then the density character of $2^{N}$ will be equal to $m$. To accomplish this we extend $\mu_{1}$ by a transfinite inductive definition. Suppose, for ordinals $\beta<\alpha, \mu_{1}$ has been extended from $\mathscr{A}_{0}$ to an algebra $\mathscr{A}_{\beta}$ so that $\mathscr{A}_{\gamma} \subset \mathscr{A}_{\beta} \subset 2^{N}$ if $\gamma<\beta$ and $\mu_{1}$ when restricted to $\mathscr{A}_{\gamma}$ from $\mathscr{A}_{\beta}$ is the extension to $\mathscr{A}_{\gamma}$ of $\mu_{1}$ from $\mathscr{A}_{0}$ and so that $\mathscr{A}_{0}$ is $d_{\mu_{1}}$-dense in $\mathscr{A}_{\beta}$ for all $\beta<\alpha$. If $\alpha$ is a limit ordinal let $\mathscr{A}_{\alpha}=\mathbf{U}_{\beta<\alpha} \mathscr{A}_{\beta}$ and let $\mu_{1}$ be the unique extension to $\mathscr{A}_{\alpha}$ whose restrictions to $\mathscr{A}_{\beta}$ are the already given extension of $\mu_{1}$ for $\beta<\alpha$. It is immediate that $\mathscr{A}_{0}$ is $d_{\mu_{1}}$-dense in $\mathscr{A}_{\alpha}$ in this case. If $\alpha$ is not limit ordinal, $\beta$ is its predecessor, and if $\mathscr{A}_{\beta} \neq 2^{N}$ select

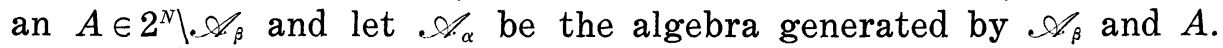
It is well known that, if $\left(\mu_{1}\right)_{*}(A)$ and $\left(\mu_{1}\right)_{*}(A)$ are the outer and inner measures of $A$ with respect to $\mu_{1}$ on $\mathscr{A}_{\beta}$, there is an extension of $\mu_{1}$ to $\mathscr{A}_{\alpha}$ with $\mu_{1}(A)=\lambda$ whenever $\left(\mu_{1}\right)_{*}(A) \leqq \lambda \leqq\left(\mu_{1}\right)_{*}(A)$. Select an extension $\mu_{1}$ so that $\mu_{1}(A)=\left(\mu_{1}\right)_{*}(A)$. It is easily deduced that $A$ is in the $d_{\mu_{1}}$-closure of $\mathscr{A}_{\beta}$ so there is a sequence $\left\{A_{n}\right\} \subset \mathscr{A}_{\beta}$ with $d_{\mu_{1}}\left(A_{n}, A\right) \rightarrow 0$. From this it follows that $d_{\mu_{1}}\left(A_{n} \cap B, A \cap B\right) \rightarrow 0$ and $d_{\mu_{1}}\left(A_{n}^{c} \cap B, A^{c} \cap B\right) \rightarrow 0$ for all $B \in \mathscr{A}_{\beta}$. Thus, $\mathscr{A}_{\beta}$ is $d_{\mu_{1}}$ dense in $\mathscr{A}_{\alpha}$. Thus, $\mathscr{A}_{0}$ is $d_{\mu_{1}}$-dense in $\mathscr{A}_{\alpha}$. For all ordinals $\alpha$ we have $\mathscr{A}_{0} d_{\mu_{1}}$ dense in $\mathscr{A}_{\alpha}$. For some ordinal $\alpha, \mathscr{A}_{\alpha}=2^{N}$. At this stage the desired extension has been accomplished.

The second step of the proof is to construct a probability measure $\mu$ on $2^{N}$ such that $2^{N}$ is $d_{\mu}$-meager with density character $m$. Let $\mu_{0}$ be the countably additive measure on $N$ with $\mu_{0}(\{n\})=$ $2^{-n}$ for $n \in N$. Let $\mu=1 / 2\left(\mu_{0}+\mu_{1}\right)$ where $\mu_{1}$ is constructed in the preceding paragraph. Since $\mu$ is strictly positive on $N$, Proposition 11 shows that $2^{N}$ is $d_{\mu}$-meager. From the construction of $\mu_{1}$ it follows that there is a set $\left\{A_{\alpha}: \alpha<m\right\}$ (where $m$ is considered the first ordinal of cardinality $m$ ) with $\mu_{1}\left(A_{\alpha} \Delta A_{\beta}\right)=1 / 2$ for $\alpha \neq \beta$. Thus, $d_{\mu}\left(A_{\alpha}, A_{\beta}\right)=\mu\left(A_{\alpha} \Delta A_{\beta}\right) \geqq(1 / 2) \mu_{1}\left(A_{\alpha} \Delta A_{\beta}\right)=1 / 4$. Thus, the density character of $2^{N}$ is at least $m$. Let $\left\{E_{\alpha}: \alpha<m\right\}$ be a $d_{\mu_{1}}$-dense set in $2^{N}$. Let $N_{f}$ be the $d_{\mu_{0}}$-dense set of finite subsets of $2^{N}$. All sets which differ from an $E_{\alpha}$ by an element of $N_{f}$ form a $d_{\mu}$-dense set of cardinality $m$. Thus, the density character of $2^{N}$ under $d_{\mu}$ is at most $m$, hence is equal to $m$. This establishes the proposition for the case $\mathscr{B}=2^{N}$.

The third step of the proof consists of extending from the case $\mathscr{B}=2^{N}$ to the case where $\mathscr{B}$ is an arbitrary complete Boolean algebra. This is done imitating arguments given in [4]. An infinite complete algebra contains an infinite disjoint sequence $\left\{A_{n}: n \in N\right\}$ 
hence contains a subalgebra isomorphic to the clopen algebra of the Alexandroff compactification, $N \cup\{\infty\}$, of $N$. There is a continuous surjection from the Stone space, $X_{\mathscr{B}}$, of $\mathscr{B}$ onto $N \cup\{\infty\}$. Thus, by results on projective covers on Gleason spaces, [3], there is a continuous surjection of $X_{\mathscr{B}}$ onto $\beta N$ the Gleason space of $N \cup\{\infty\}$. Consequently, by results in [4], there is a closed subspace $Y$ of $X_{\mathscr{A}}$ on which the surjection from $X_{\mathscr{O}}$ to $\beta N$ is a homeomorphism. The closed set $Y$ is the Stone space of the algebra $\mathscr{B} / \mathscr{I}$ where $\mathscr{I}$ is some ideal of $\mathscr{B}$. Thus, there is a Boolean isomorphism $j: \mathscr{B} / \mathscr{I} \rightarrow$ $2^{N}$. Let $\mu$ denote both the measure constructed in the previous paragraph on $2^{N}$ and its pull back under $j$ to $\mathscr{B} / \mathscr{F}$. Let $\mu$ also denote the measure on $\mathscr{B}$ obtained by defining $\mathscr{J}$ to consist of $\mu$ negligible sets. $\mathscr{B} / \mathscr{F}=\mathscr{P}_{\mu}$ is $d_{\mu}$-meager and has density character $m$. This complete the proof of the proposition.

REMARKS. (1) This result is best possible in that on $2^{N}$ any measure $\mu$ yields density character at most the cardinality, $2^{\aleph_{0}}$, for $2^{N}$.

(2) Can higher cardinals be obtained for $d_{\mu}$-density character of sufficiently large complete Boolean algebras $\mathscr{B}$ with $\mathscr{B}_{\mu} d_{\mu^{-}}$ meager?

(3) There is no hope, by Proposition 2, that $\mu$ can be constructed in a countably additive fashion. This is because $\mathscr{B}_{\mu}$ as the quotient of a complete algebra by an ideal is an $F$-algebra, [4], which satisfies the countable chain condition hence is complete.

(4) The measure $\mu$ constructed in Proposition 16 is non-atomic. Candeloro and Sacchetti, [10] in the proof of Theorem 2.4 show that if $\mathscr{B}$ is $2^{X}$ and $\mu$ is non-atomic there is a $\sigma$-algebra $\mathscr{A}$ of subsets of $X$ such that $\mathscr{A}$ under $d_{\mu}$ is homeomorphic to $\{0,1\}^{N}$. Thus, $\mathscr{B}_{\mu}$ while $d_{\mu}$-meager is fairly large.

(5) Seever in [26] shows that the Vitali-Hahn-Saks theorem is valid for finitely additive measures on $\mathscr{B}_{\mu}$ if $\mathscr{B}_{\mu}$ is $\sigma$-complete. Labuda, [17], shows that the Vitali-Hahn-Saks theorem is true when $\mathscr{B}_{\mu}$ isn't $d_{\mu}$-meager. Propositions 15 and 16 demonstrates the independence of their results.

\section{REFERENCES}

1. T. Armstrong, Borel measures on compact groups are meager, Illinois J. Math., (to appear).

2. - Gleason spaces and topological dynamics, Indiana J. Math., 29 (1980), 737-746.

3. T. Armstrong and K. Prikry, Residual measures, Illinois J. Math., 22 (1978), 64-78. 4. - Liapounoff's theorem for non-atomic, finitely additive, finite dimensional, vector valued measures, Trans. Amer. Math. Soc., (to appear).

5. W. C. Bell, A decomposition of additive set functions, Pacific J. Math., 72 (1977), 305-311. 
6. W. C. Bell, Hellinger integrals and set function derivatives, Houston J. Math., 5 (1979), 465-481.

7. —, Approximate Hahn decompositions, uniform absolute continuity and uniform integrability, J. Math. Anal. Appl., (to appear).

8. S. Bochner and R. S. Phillips, Additive set functions and vector lattices, Ann. Math., 42 (1941), 316-325.

9. V. Bogdan and R. A. Oberle, Topological rings of sets and the theory of vector measures., Dissert. Math., 154 (1978).

10. D. Candeloro and A. M. Sacchetti, Su alcuni problemi relativi a misura scalari sub additive e applicazionial caso dell'additivita finita., Atti. Sem. Mat. Fis. Univ. Modena, 27 (1978), 284-296.

11. S. Cobzas, Hahn decompositions of finitely additive measures, Arch. Math., 27 (1976), 620-621

12. J. Diestel and J, J. Uhl, Jr., Vector Measures, AMS Math. Surveys, 15, Providence, 1977.

13. L. Drewnowski, Equivalence of Brooks-Jewett, Vitali-Hahn-Saks, and Nikodym Theorems, Bull. Acad. Polon. Sci. Ser. Sci. Math. Astronom. Phys., 20 (1972), 725-731.

14. L. Dubins and L. J. Savage, Inequalities for Stochastic Processes. How to Gamble if You Must, Dover, New York, 1976.

15. P. Halmos, Measure Theory, Van Nostrand, Princeton, 1950.

16. J. Kelley, General Topology, Van Nostrand, Princeton, 1955.

17. I. Labuda, Sur quelques generalizations des theoremes de Nikodym et de VitaliHahn-Saks, Bull. Acad. Polon. Sci. Ser. Sci. Math. Astronom. Phys., 20 (1972), 447-456. 18. D. Maharam, Category, Boolean algebras and measure. General topology and its relation to modern analysis and algebra, Springer, New York, (1977), 124-135.

19. H. Maynard, A Radon-Nikodym theorem for finitely additive measures, Pacific J. Math., 83 (1979), 401-413.

20. J. C. Oxtoby, Spaces that admit a category measure, J. Reine Angew. Math., 205 (1961), 156-170.

21. J. C. Oxtoby, Cartesion products of Baire spaces, Fund. Math., 49 (1961), 157-166.

22. - Measure and Category, Springer, New York, 1971.

23. D. Plachky, Extremal and monogenic additive set functions, Proc. Amer. Math. Soc., 54 (1976), 193-196.

24. M. Bhaskara and K. P. S. Bhaskara Rao, Existence of non-atomic charges, J. Australian Math. Soc., 25 (1978), 1-6.

25. - Topological properties of charge algebras, Rev. Roumaine Math. Pures Appl., 22 (1977), 363-375.

26. G. L. Seever, Measures on F-spaces, Trans. Amer. Math. Soc., 133 (1968), 267-280.

27. Z. Semademi, Banach Spaces of Continuous Functions I, Polish Scientific, Warsaw, 1971.

28. R. Sikorski, Boolean Algebras, Springer, New York, 1964.

29. A. Sobczyk and P. C. Hammer, $A$ decomposition of additive set functions, Duke Math. J., 11 (1944), 839-840.

30. - The ranges of additive set functions, Duke Math. J., (1944), 847-851.

Received December 9, 1980 and in revised form May 20, 1981.

NORTHERN ILLINOIS UNIVERSITY

DEKaLB, IL 60115 


\title{
PACIFIC JOURNAL OF MATHEMATICS
}

\section{EDITORS}

\author{
DONALD BABBITT (Managing Editor) \\ University of California \\ Los Angeles, CA 90024 \\ Hugo RossI \\ University of Utah \\ Salt Lake City, UT 84112 \\ C. C. MOORE and Arthur Agus \\ University of California \\ Berkeley, CA 94720
}

\section{J. DugundjI}

Department of Mathematics

University of Southern California

Los Angeles, CA 90007

R. FinN and J. Milgram

Stanford University

Stanford, CA 94305

\section{ASSOCIATE EDITORS}
R. ARENS
E. F. BeCKENBACH
B. H. NeumanN
F. WOLF
K. YoshidA

\section{SUPPORTING INSTITUTIONS}

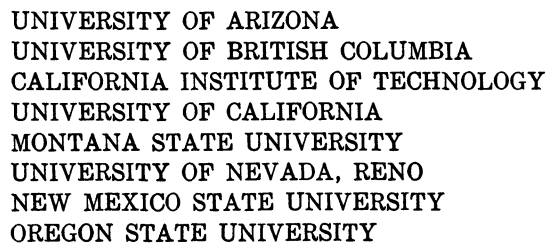

UNIVERSITY OF ARIZONA

UNIVERSITY OF BRITISH COLUMBIA

CALIFORNIA INSTITUTE OF TECHNOLOGY

UNIVERSITY OF CALIFORNIA

MONTANA STATE UNIVERSITY

UNIVERSITY OF NEVADA, RENO

NEW MEXICO STATE UNIVERSITY

OREGON STATE UNIVERSITY

\author{
UNIVERSITY OF OREGON \\ UNIVERSITY OF SOUTHERN CALIFORNIA \\ STANFORD UNIVERSITY \\ UNIVERSITY OF HAWAII \\ UNIVERSITY OF TOKYO \\ UNIVERSITY OF UTAH \\ WASHINGTON STATE UNIVERSITY \\ UNIVERSITY OF WASHINGTON
}

The Supporting Institutions listed above contribute to the cost of publication of this Journal, but they are not owners or publishers and have no responsibility for its content or policies.

Mathematical papers intended for publication in the Pacific Journal of Mathematics should be in typed form or offset-reproduced, (not dittoed), double spaced with large margins. Please do not use built up fractions in the text of the manuscript. However, you may use them in the displayed equations. Underline Greek letters in red, German in green, and script in blue. The first paragraph or two must be capable of being used separately as a synopsis of the entire paper. Please propose a heading for the odd numbered pages of less than 35 characters. Manuscripts, in triplicate, may be sent to any one of the editors. Please classify according to the scheme of Math. Reviews, Index to Vol. 39. Supply name and address of author to whom proofs should be sent. All other communications should be addressed to the managing editor, or Elaine Barth, University of California, Los Angeles, California, 90024.

50 reprints to each author are provided free for each article, only if page charges have been substantially paid. Additional copies may be obtained at cost in multiples of 50 .

The Pacific Journal of Mathematics is issued monthly as of January 1966. Regular subscription rate: $\$ 102.00$ a year (6 Vols., 12 issues). Special rate: $\$ 51.00$ a year to individual members of supporting institutions.

Subscriptions, orders for numbers issued in the last three calendar years, and changes of address shoud be sent to Pacific Journal of Mathematics, P.O. Box 969, Carmel Valley, CA 93924, U.S.A. Old back numbers obtainable from Kraus Periodicals Co., Route 100, Millwood, NY 10546.

\section{PUBLISHED BY PACIFIC JOURNAL OF MATHEMATICS, A NON-PROFIT CORPORATION}

Printed at Kokusai Bunken Insatsusha (International Academic Printing Co., Ltd.). 8-8, 3-chome, Takadanobaba, Shinjuku-ku, Tokyo 160, Japan. 


\section{Pacific Journal of Mathematics}

Vol. 99, No. $2 \quad$ June, 1982

Thomas E. Armstrong and Karel Libor Prikry, On the semimetric on a

Boolean algebra induced by a finitely additive probability measure . . . .249

Walter Russell Bloom, Strict local inclusion results between spaces of

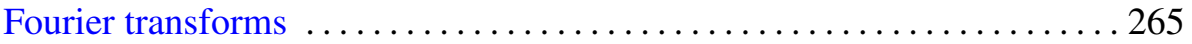

Richard Clark Brown, Notes on generalized boundary value problems in

Banach spaces. II. Infinite-dimensional extension theory ........... 271

Sui Sun Cheng, Isoperimetric eigenvalue problem of even order differential

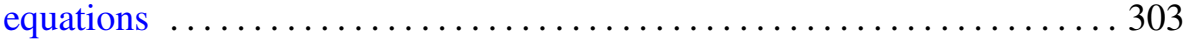

Lung O. Chung and Jiang Luh, Derivations of higher order and

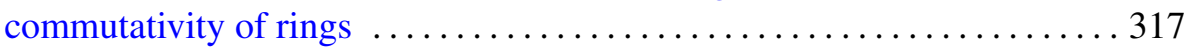

Ali Ahmad Fora, A fixed point theorem for product spaces . . . . . . . . . 327

Barry J. Gardner, Radical classes of regular rings with Artinian primitive

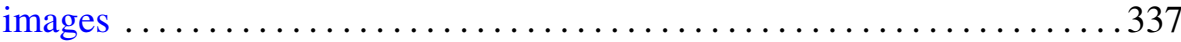

John Brady Garnett and Peter Wilcox Jones, BMO from dyadic BMO . . . 351

Allen E. Hatcher, On the boundary curves of incompressible surfaces . . . . 373

Richard Howard Hudson and Kenneth S. Williams, Resolution of

ambiguities in the evaluation of cubic and quartic Jacobsthal sums .....379

Viktor Losert, Counter-examples to some conjectures about doubly

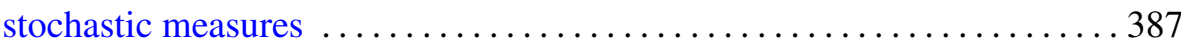

Kenneth Derwood Magill, Jr., P. R. Misra and Udai Bhan Tewari,

Structure spaces for sandwich semigroups

Mark Mandelker, Continuity of monotone functions

Kenneth Guy Miller, An index theorem and hypoellipticity on nilpotent Lie groups ......................................... 419

Evelyn M. Nelson, Homomorphisms of mono-unary algebras . . . . . . . . . 427

Marvin E. Ortel, The support of an extremal dilatation . . .

R. S. Pathak and O. P. Singh, Finite Hankel transforms of distributions . . . 439

Richard Cole Penney, The theory of ad-associative Lie algebras

Linda Ruth Sons, Zero distribution of functions with slow or moderate

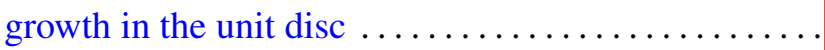

Russell Bruce Walker, Transversals to laminations 